\title{
Koronare Herzkrankheit als häufigste Ursache Plötzlicher Herztod im Sport
}

\author{
H. Löllgen, R. Gerke, S. Lenz, Sana-Klinikum Remscheid
}

NOTFALLMEDIZIN 2003, 29: 148-158

Ein plötzlicher Herztod im Sport ist immer ein dramatisches Ereignis, die Abklärung häufig unbefriedigend. Neuere Krankheitsbilder erklären Zwischenfälle, die früher als idiopathisch gegolten haben. Bei jüngeren Sportlern überwiegen angeborene strukturelle Herzkrankheiten. Bei älteren Sportlern, solchen über 35 Jahren, steht die koronare Herzkrankheit als Ursache ganz im Vordergrund. Mit der Entwicklung automatischer Defibrillatoren stehen wirksame Maßnahmen, auch in der Hand geschulter Laien, zur Behandlung und Verhinderung des plötzlichen Herztodes zur Verfügung. Größere Bedeutung aber kommt der Prävention zu. Eine gezielte und standardisierte Befragung ist die wichtigste Maßnahme, eine qualifizierte sportärztliche Untersuchung vermag das Risiko weiter zu mindern.

$\mathrm{P}$ lötzliche Todesfälle im Sport finden meist großes Interesse in der Bevölkerung und den Medien. Man geht davon aus, dass Sportler durch ihr regelmäßiges Training seltener von plötzlichen Todesfällen betroffen sind als die Allgemeinbevölkerung. Beobachtungen, Fallbeschreibungen, Reihenuntersuchungen und neue wissenschaftliche Ergebnisse kardiovaskulärer Untersuchungen in den letzten Jahren haben neue Erkenntnisse zu dieser Thematik, aber auch zur Vorbeugung ergeben. Im Vordergrund steht eine sorgfältige Anamnese, im Einzelfall vermag eine abgestufte sportärztliche Vorsorgeuntersuchung das Risiko weiter zu mindern.

Üblicherweise versteht man unter einem plötzlichen Herztod den unerwarteten Eintritt eines kardiovaskulär bedingten Bewusstseinsverlustes mit Tod innerhalb einer Stunde. Andere Definitionen berücksichtigen auch den Tod innerhalb von zwölf oder 24 Stunden nach Auftreten der Symptome als plötzlichen Herztod. Meist tritt beim Sportler der Tod während oder nach der körperlichen Belastung ein, plötzliche Todesfälle sind bei Sportlern auch im Schlaf oder während Körperruhe möglich. Man unterscheidet daher den plötzlichen Tod beim Sportler im oder ohne Zusammenhang mit sportlicher Aktivität (Tab. 1, Abb. 2). Die nähere Charakterisierung des Todesfalles ist mitunter schwierig, da eine Autopsie nicht immer die Ursache sichern kann (z.B. WPW-Syndrom, langes QT-Syndrom). Ferner muss zwischen hochtrainierten, jüngeren Sportlern sowie Breiten- und/oder Gelegenheitssportlern höheren Alters unterschieden werden. 


\section{Abb. 1 Ursachen kardialer Zwischenfälle im Sport in Abhängigkeit vom Alter}
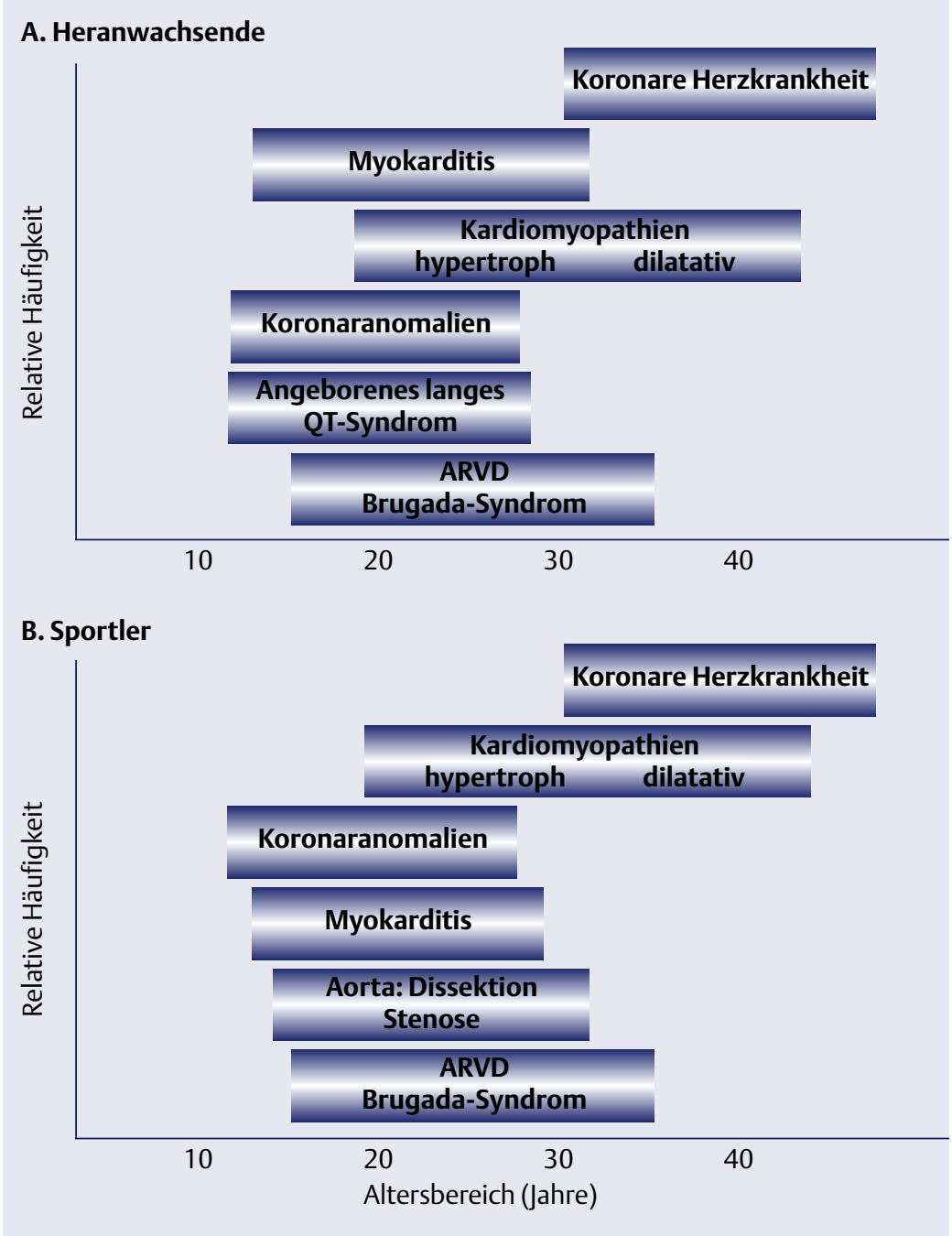

\section{Häufigkeit}

Zur Häufigkeit des plötzlichen Herztodes liegen viele Studien vor, diese sind aber selten prospektiv angelegt und erfassen nicht immer alle Todesfälle (Tab. 1). Viele Studien sind retrospektiv durchgeführt oder basieren auf Analysen von Versicherungsstatistiken. Der plötzliche Tod in der Allgemeinbevölkerung liegt in etwa bei 1-2 Todesfällen pro 1000 Einwohnern. Bei Personen unter 30 Jahren liegt die Rate bei 0,5 bis 1 auf 100000 (22), davon finden $8 \%$ während Belastungen statt. Bei amerikanischen Schülern rechnet man mit einem Todesfall auf 200000 bis 300 000/Jahr. In einigen Studien liegt die Häufigkeit etwas höher ( 2 bis 7/100 000). Bei Studenten soll die Rate bei 1 bzw. 5 pro 70000 Per- sonen pro Jahr liegen $(4,12,22,32)$. Frauen sind mit einem Anteil von $10 \%$ deutlich seltener betroffen. Afro-Amerikaner sind häufiger betroffen, da bei ihnen eine hypertrophe Kardiomyopathie häufiger vorkommt. Belastungsinduzierte Todesfälle bei Herzkranken treten demgegenüber häufiger auf, zwischen 30 und $40 \%$ der akuten Todesfälle bei Herzkranken treten im Zusammenhang mit körperlicher Belastung auf. Allerdings sind die Meinungen hierzu widersprüchlich, einige Autoren konnten keinen Zusammenhang des plötzlichen Todes mit der Sportausübung nachweisen $(6,32)$. Verschiedene Übersichtsarbeiten ergeben aber einen Zusammenhang mit einer durchschnittlichen Todesrate von 15\% bei körperlicher Belastung.
Diese Zahl betrifft alle Personen, also Trainierte, Untrainierte und Herzkranke. Bei Marathonläufern tritt ein plötzlicher Tod während des Laufes seltener auf als in der Allgemeinbevölkerung.

Aus der kritischen Analyse begünstigen folgende Faktoren das Auftreten eines plötzlichen Todes im Sport:

- Vorbestehende kardiale Grundkrankheit (bekannt oder unbekannt),

- höheres Alter (über 40 Jahre) mit einem Gipfel zwischen 40 und

- Neubeginn einer sportlichen Aktivität mit plötzlichem und $\mathrm{zu}$ intensivem Beginn $(12,14,25)$.

\section{Ursachen}

Plötzliche Todesfälle im Sport, vor allem bei scheinbar gesunden jüngeren Sportlern, beruhen fast immer auf einer organischen kardialen Grundkrankheit (Abb. 1, Tab. 1). Be-

\section{Tab. 1 Ursachen eines plötzlichen Herztodes im Sport}

Alter unter $\mathbf{3 5}$ Jahre:

- Kardiomyopathie, dilatative

- Kardiomyopathie, hypertrophe

- Koronare Anomalie

- Linkshypertrophie

- Aortenruptur

- Muskelbrücke des RIVA*

- Aortenklappenstenose

- Myokarditis

- ARVD

- Mitralklappenprolaps

- Koronare Herzkrankheit

- Sonstiges

Alter über 35 Jahre:

- Koronare Herzkrankheit

- Kardiomyopathie

- Herzklappenerkrankung (Aortenstenose, -insuffizienz)

- Selten: Myokardiale Ischämie bei Spasmus, Muskelbrücke

- Ventrikuläre dysplastische Syndrome: arrhythmogene rechtsventrikuläre, Dysplasie, Brugada-Syndrom, langes QT-Syndrom

- Gefäßanomalien: Marfan-Syndrom

- Mitralklappenprolaps (?)

- Unerkannte akzessorische Leitungsbahnen

(modifiziert nach Pellicia (23) und Maron (17).)

${ }^{*}$ RIVA: Ramus interventrikularis anterior

+ARVD: Arrhythmogene rechtsventrikuläre Dysplasie 
merkenswert ist, dass in den USA bei den unter 35-jährigen die HOCM (hypertrophische obstruktive Cardiomyopathie) überwiegt, in Venedig hingegen die ARVD (arrhythmogene rechtsventrikuläre Dysplasie) $(5,18)$. Dies beruht möglicherweise auf dem speziellen Interesse der Kardiologen und Sportärzte in Venedig an der rechtsventrikulären Erkrankung und an der gesteigerten Aufmerksamkeit an diesem Krankheitsbild in den letzten Jahren. Zum anderen dürfte die Mehrzahl der Sportler mit hypertropher Kardiomyopathie frühzeitig erkannt worden sein und intensiven Sport nicht mehr betreiben. Die Regio Veneto ist

\section{Abb. 2 Pathophysiologische Mechanismen}

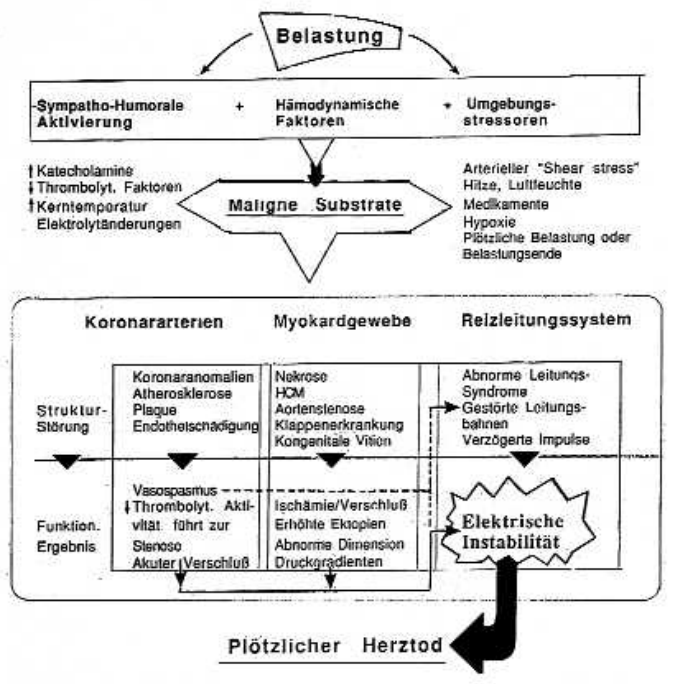

(Quelle: Myerburg)

\section{Tab. 2 Passagere Risikofaktoren für Arrhythmien und plötzlichen Herztod im Sport}

Systemische Faktoren

- Hämodynamische Dysfunktion

- Hypoxämie, Azidose

- Elektrolytstörungen

Neurophysiologische Faktoren

- Zentral, systemisch

- Lokal-kardial (Transmitter, Rezeptor)

Toxisch kardialer Effekt

- Idiosynchrasie

- Proarrhythmie

- Passagere Proarrhythmie

(Nach Myerburg,1998) im Übrigen eine der wenigen Gegenden, wo jedermann sich sportärztlich untersuchen lassen kann (5, 7, 17, 18, 32). Bei älteren Menschen und Breitensportlern überwiegt in hohem Prozentsatz die koronare Herzkrankheit als Ursache des plötzlichen Herztodes (Tab. 1, Abb. 1).

Neben der ARVD hat in den letzten Jahren auch das Brugada-Syndrom und das lange QT-Syndrom Aufmerksamkeit bei Todesfällen im Sport erregt (Tab. 4, 5) (1, 2, 8, 24). Beide Erkrankungen erklären möglicherweise auch frühere Beschreibungen von Sporttodesfällen ohne erkennbare Ursachen (so genanntes idiopathisches Kammerflimmern). Hierzu könnte auch das WPWSyndrom (Wolff-Parkinson-WhiteSyndrom) mit intermittierendem Vorhofflimmern gezählt werden. Rein arrhythmogene Todesfälle ohne organisches Substrat sind naturgemäß postmortal meist nicht nachweisbar. Molekularbiologische Analysen ermöglichen heute eine Diagnose beim Lebenden, wenn ein entsprechender Verdacht besteht (26). Eine familiäre Häufung ist möglich, vor allem bei den dilatativen und hypertrophen Kardiomyopathien (Abb. 3) sowie bei ARVD und BrugadaSyndrom, aber auch beim langen QT-Syndrom (26, 27). Das Syndrom der langen QT-Dauer kann entweder angeboren auftreten mit ungünstiger Prognose oder erworben sein. Beim erworbenen QT-Syndrom ist vor allem auf medikamentöse Auslöser zu achten wie Antibiotika (Makrolide) oder Antihistaminika und andere Substanzgruppen (Tab. 5).

Entzündliche Herzerkrankungen können ebenfalls zum plötzlichen Tod führen, sie sind aber seltener als früher vermutet. Selten kommen Borreliose oder kardiale Sarkoidose als Ursache infrage. Schließlich können stumpfe Thoraxtraumata mit präkordialer Einwirkung Rhythmusstörungen auslösen, und zwar sowohl Vorhofflimmern als auch Kammerflimmern (sog. chest blunt) (10, 15). Eine solche commotio cordis wird selten nach Einwirkung eines Sportballes (Kricket, Baseball), eines Pucks im Eishockey oder eines Stoßes mit dem Arm oder Ellenbogen beschrieben $(10,15)$.
Eine weitere seltene Ursache ist das Marfansyndrom (Tab. 6). Bei auffallender Körperlänge (über $190 \mathrm{~cm}$, z.B. bei Ruderern, Basketballspielern) sollte auf Marfansyndrome geachtet werden, abortive Formen sind möglich. Todesursache ist meist eine Aortenruptur. Schließlich können allgemeine passagere Veränderungen auftreten, die Rhythmusstörungen begünstigen können (Tab. 2).

\section{Pathophysiologie}

Auslösend für einen Zwischenfall und plötzlichen Tod im Sport ist meist eine akute, komplexe ventrikuläre Arrhythmie, seltener eine Ischämie. Grundsätzlich können auch andere Rhythmusstörungen fatale Folgen haben, dann aber meist mit sekundären nicht-kardialen Ursachen wie beispielsweise Unfall oder Absturz (Abb. 2).

Rhythmusstörungen im Sport lassen sich einteilen in:

- symptomatisch oder asymptomatisch

- benigne oder maligne

- mit oder ohne nachweisbare strukturelle Herzerkrankung.

Symptomatische und asymptomatische Arrhythmien können benigne oder maligne sein. Auch erlaubt die Symptomatik keinen Rückschluss auf eine mögliche Gefährdung.

Arrhythmien können Folge struktureller kardialer Erkrankungen sein oder Folge passagerer neurohumoraler Veränderungen $(2,3,13,14)$ (Tab. 2). Die Arrhythmie wird dann manifest, wenn zusätzliche, nicht selten belastungsinduzierte begünstigende Faktoren hinzutreten. Ein wesentlich bestimmender Faktor für Arrhythmien während Belastungen ist die Zunahme des Sympathikotonus und die Abnahme des Vagotonus mit einer entsprechenden Verschiebung des Gleichgewichtes beider nervaler Anteile. Bei standardisierter Belastung (Ergometrie) mit fortlaufender EKG-Registrierung sind Arrhythmien bei Gesunden seltener als bei Patienten mit struktureller Herzkrankheit. Supraventrikuläre Tachykardien und intermittierendes Vorhofflimmern kommen in rund 1 bis 3\% bei Gesunden, im Vergleich 


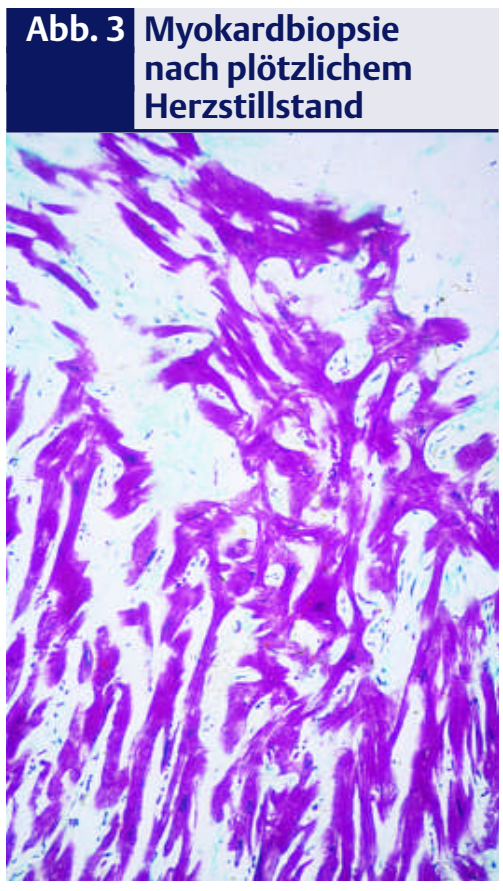

Plötzlicher Herzstillstand bei einem 19-jährigen Freizeitfußballer auf dem Platz. Erfolgreiche Reanimation, Tod nach hypoxischem Hirnschaden. Die Histologie zeigt einen Umbau der Myozyten durch Bindegewebe, die Muskelfasern sind in ihrer Textur zerstört.

(Überlassen durch Prof. Sandritter, Freiburg)

zu 27\% bei Patienten vor, supraventrikuläre Extrasystolen sind etwas häufiger (ca. 20\% bei Gesunden) (13, 14). Ventrikuläre Arrhythmien werden in 1 bis 39\% bei Gesunden registriert im Vergleich zu 20-80\% bei Patienten.

\section{Art der Rhythmusstörung}

Generell lassen sich Arrhythmien in der klassischen Weise einteilen in supraventrikuläre und ventrikuläre sowie bradykarde und tachykarde Formen. Zu beachten ist, dass beim trainierten Sportler manche - vor allem bradykarde - Rhythmusstörungen Normvarianten sind (Tab. 3) (23). Auch die Ätiologie dieser Normvarianten beruht auf der trainingsbedingten Änderung der autonomen Funktion mit einem Überwiegen des Vagotonus. Es sei betont, dass diese EKG-Beobachtungen bei Normalpersonen krankhaft sein können, beim Sportler aber physiologisch und reversibel sind, also nicht behandelt zu werden brauchen. Die Abgrenzung ist mitunter schwierig.

\section{Supraventrikuläre bradykarde Arrhythmien}

Eine Sinusbradykardie bei trainierten Sportlern ist ein häufiger und typischer Befund. Der normale Frequenzanstieg unter Belastung weist auf die physiologische Anpassung hin, eine Therapie ist nicht erforderlich. Ebenso sind AV-Block ersten sowie zweiten Grades (Typ Wenckebach) trainingsbedingte Anpassungserscheinungen und ohne Konsequenz, hier kann Sport ohne Einschränkung betrieben werden. Ein AV-Block zweiten Grades (Typ Mobitz) ist seltener und bedarf der Kontrolle. Bei normaler Belastungsreaktion wird man ebenfalls abwarten. Ein drittgradiger angeborener AV-Block wird gelegentlich bei Sporttreibenden beobachtet, eine Therapie ist nur bei eindeutiger Symptomatik indiziert (Schrittmacher). Der erworbene totale AV-Block ist beim Sportler selten und bedarf der weiteren Abklärung, wobei je nach Anamnese (Zeckenbiss) auch an eine Borreliose gedacht werden muss. Bradykarde Arrhythmien führen selten zum Tode, es sei denn sie treten bei einer Sportart auf, bei der ein Kollaps zu einer akuten Gefährdung führt, wie bei Fallschirmsprin- gen, Segelfliegen, Schwimmen im Meer oder beim Tauchen. Ähnliches gilt für supraventrikuläre tachykarde Arrhythmien.

\section{Supraventrikuläre tachykarde Arrhythmien}

Selten, aber von Bedeutung sind paroxysmale supraventrikuläre Tachykardien, vor allem AV-Nodale Reentry-Tachykardien und das WPWSyndrom. AV-Nodale Arrhythmien können gelegentlich zu Kollaps und nachfolgendem Tod führen. Von besonderer Bedeutung aber ist das WPW-Syndrom mit intermittierendem Vorhofflimmern (Abb. 4).

Bei WPW-Tachykardien und zusätzlichem intermittierendem Vorhofflimmern tritt eine vitale Gefährdung des Sportlers dann ein, wenn die Vorhofaktionen rasch (1:1) über-

\section{Tab. 3 Normvarianten im EKG bei Sportlern}

- Sinusbradykardie

- Sinusarrhythmie

- Inkompletter Rechtsschenkelblock

- Vorhofleitungsstörungen (P-Welle)

- Links- und Rechtshypertrophie nach Spannungskriterien

- ST-Hebungen oder ST-Senkungen

- QT-Dauer im oberen Grenzbereich

- Schmale vergrößerte oder negative T-Wellen

\section{Tab. 4 Diagnostische Hinweise auf Arrhythmogene rechtsventrikuläre Dysplasie (ARVD) und Brugada-Syndrom}

ARVD

- Globale oder regionale rechtsventrikuläre Dysfunktion

- Histologie: Fibrolipomatose (Typ 1, 2)

- Repolarisationsstörungen:

T-Inversion in den rechtspräkordialen Ableitungen

Epsilonpotential oder QRS > $110 \mathrm{~ms}$

positive Spätpotentiale

- Form der Arrhythmien : Kammertachykardien (monomorph) oder gehäufte VES

- Familiäre Belastung

Brugada-Syndrom

- Primär abnorme elektrophysiologische Aktivität im rechten Ventrikel (Frühform der ARVD?)

- Familiäre Veranlagung (lonenkanaldefekt)

- EKG: Rechtsschenkelblockbild, hoher Abgang der ST-Strecke mit deszendierend verlaufender ST-Hebung in V1-V3 mit negativem T, evtl. Knotung im deszendierenden Teil von QRS

(Quelle: Wilde, 2002) 
geleitet wird und funktionell einer Kammertachykardie beziehungsweise einem Kammerflimmern entspricht. Hier ist der Hinweis wichtig, dass eine kausale Therapie mit Katheterablation zu einer Heilung führen kann. Bei symptomatischen Sportlern mit WPW-Syndrom und/ oder nachweisbaren Tachykardien wird man heute eher großzügig eine solche Ablation ins Auge fassen.

\section{Ventrikuläre Arrhythmien}

Ventrikuläre Extrasystolen bei Sportlern sind meist harmloser Natur. Selten beobachtet man einen lang andauernden Bigeminus mit Ursprung im rechtsventrikulären Ausflusstrakt, wodurch eine hämo-

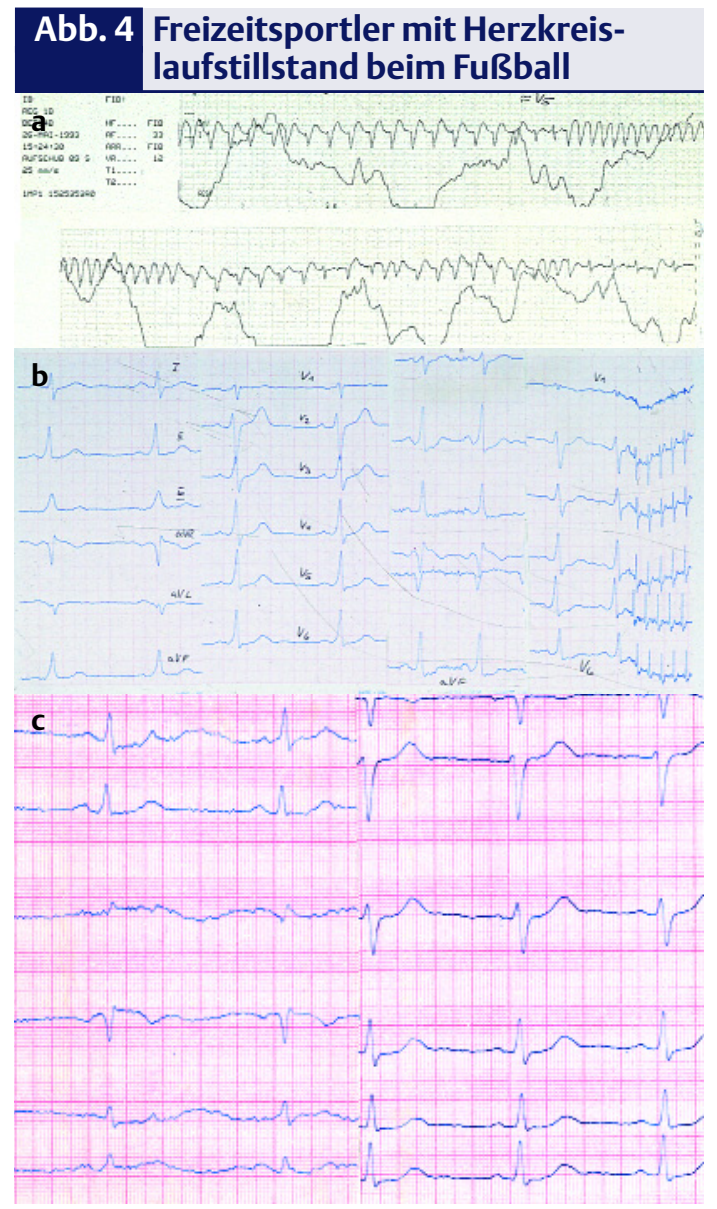

Erfolgreiche Reanimation nach Herzkreislaufstillstand. Bei Aufnahme Kammerflimmern, Defibrillation (a). Später Vorhofflimmern, nach Kardioversion Sinusrhythmus mit typischem WPW-Syndrom (b). Bei unauffälligen Koronargefäßen erfolgte eine erfolgreiche Ablation des akzessorischen Bündels (Prof. Breithardt, Münster). Anschließend regulärer Rhythmus (c). dynamische Beeinträchtigung auftreten kann. Diese Arrhythmie kann durch eine Katheterablation beseitigt werden. Die Indikation besteht bei hämodynamischer Beeinträchtigung und ausgeprägter Minderung der Lebensqualität oder bei starkem Leidensdruck.

Plötzliche Todesfälle im Sport sind meist Folge einer komplexen ventrikulären Arrhythmie. $\mathrm{Zu}$ den malignen Arrhythmien, also solchen mit potentiell vitaler Gefährdung, auch für Sportler, gehören:

- WPW-Syndrom mit intermittierendem Vorhofflimmern

- Kammertachykardien

- Kammerflattern und Kammerflimmern

- Atypische Kammertachykardien oder Torsades de pointes (speziell bei QT-Syndrom) (Tab. 5, Abb. 6, 7).

Eine Grundkrankheit sollte bei Auftreten solcher Arrhythmien stets nachgewiesen oder ausgeschlossen werden, gegebenenfalls auch invasiv mittels Herzkatheter. Die mitunter intermittierend auftretenden Zeichen einer ARVD oder eines Brugada-Syndroms sind immer in die Differentialdiagnostik mit einzubeziehen, ebenso wie eine lange QT-Dauer im Oberflächen-EKG. Abzuklären sind weiter ein arterieller Hochdruck, hypertrophe Myokarderkrankungen (Abb. 7), eine koronare Herzkrankheit (Abb. 5), Vitien sowie entzündliche Herzkrankheiten.

\section{Akute hämodynamische}

Ursachen eines Herzstillstands

Sehr viel seltener als Arrhythmien tritt eine Ischämie (Infarkt) oder ein klappenbedingter Kollaps als Grund eines Herzstillstandes auf. Bei einem der letzten Marathonläufe in Berlin kam es bei einem Patienten mit (bekannter) Aortenklappenstenose zum Herzstillstand (pers. Mitt., Dr. Hepe). Auch Personen mit dilatativer Kardiomyopathie oder Vorhofflimmern können über lange Zeit sehr leistungsfähig sein (Abb. 3). Dennoch ist eine akute Dekompensation bei starker körperlicher Belastung möglich, ebenso wie bei entzündlichen Herzkrankheiten einschließlich einer Perikarditis.
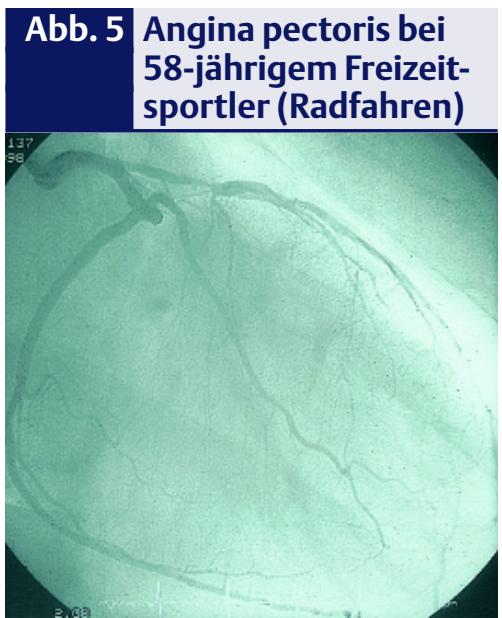

Radfahrer mit wöchentlicher Trainingsstrecke bis zu 300 km, früher mehrmaliger Teilnehmer der Tour de France. Typische Angina pectoris beim Training nach 100 bis $120 \mathrm{~km}$. Im Belastungs-EKG beim Hausarzt bis 125 Watt regelrechter Befund, bei 275 Watt in unserer Klinik eindeutige Ischämiezeichen. Hochgradige RIVA-Stenose, erfolgreiche Dilatation und Stentimplantation. Vier Wochen später (gegen ärztlichen Rat) Teilnahme an den Seniorenweltmeisterschaften.

Der akute Infarkt ist auch beim Sport möglich. Hier ist zu beachten, dass viele Sportler die Symptome einer koronaren Herzkrankheit häufig fehldeuten. Eine nicht immer typische Angina pectoris wird auf „Rückenbeschwerden“ zurückgeführt, gelegentlich auch auf Schulterschmerzen (Abb. 5). Nicht selten werden daher Sportler vom Orthopäden betreut, bevor es zum tödlichen Ereignis kommt. Hier spielt vor allem das „Ignorieren“ der kardialen Warnzeichen eine wichtige Rolle. Ausdauersportler fühlen sich „immun“ gegen eine koronare Herzkrankheit. Eigene Beobachtungen zeigen aber, dass auch bei gut trainierten Ausdauersportlern ohne Risikofaktoren eine koronare Herzkrankheit bedingt durch genetische Veranlagung auftreten kann.

In der Diskussion plötzlicher „kardiovaskulärer“ Zwischenfälle oder Todesfälle sollte beachtet werden, dass auch nicht-kardiale Ursachen vorliegen können. Schließlich ist die Einnahme von Substanzen 
oder Medikamenten - die aus verschiedenen Gründen nicht mitgeteilt wird - als weitere Ursache kardialer Zwischenfälle in Betracht zu ziehen (Tab. 7 oder Abb. 7).

Ungeklärt ist nach wie vor, ob eine erschöpfende körperliche Belastung per se, beispielsweise ein Triathlon, eine Gefährdung darstellen kann. Einige Autoren beschreiben in solchen Situationen eine „kardiale Erschöpfung“, jedoch sind bisher keine Befunde mitgeteilt worden, wonach diese Beobachtungen tatsächlich eine Gefährdung darstellen. Die sportärztliche Praxis bei Marathon- und Triathlonwettbewerben spricht gegen eine solche Gefährdung. Allerdings bleibt $\mathrm{zu}$ berücksichtigen, dass auch eine qualifizierte sportärztliche Vorsorgeun- tersuchung spätere Zwischenfälle nicht hundertprozentig ausschließen kann. Ferner nehmen auch „Personen" mit Herzkrankheiten an Ausdauerwettbewerben teil. So wird geschätzt, dass beim Berlin-Marathon bei rund 30000 Teilnehmern etwa 600 bis 800 Personen beteiligt sind, bei denen eine Herzkrankheit vorliegt und meist den Teilnehmern auch bekannt ist (Dr. Heepe, persönliche Mitteilung).

\section{Diagnostik}

Die Untersuchung von sporttreibenden Leistungsportlern wie Breiten- oder Alterssportlern basiert wie jede Untersuchung - auf Anamnese, klinischem Untersuchungsbefund und einer apparativen Diagnostik. Alle Autoren weisen in diesem

\section{Tab. 5 Ursachen eines erworbenen langen QT-Syndroms}

- Antiarrhythmika (Klasse IA-C, III u.a.)

- Antidepressiva (trizyklisch)

- Antidiabetika (Glibenclamid)

- Antimykotika (Itra-, Ketaconazol)

- Antihistaminika (Astemizol, Terfenadin)

- Antibiotika (Erythromycin, Trimethroprim, Chloroquin, Halofantrine)

- Elektrolytstörungen

- Neuroleptika (Phenothiazin)

- Prokinetika (Cisaprid)

- Selten: Subdurale Blutungen, schwere hormonelle Störungen

(Übersicht: Haferkamp, Dtsch-Ärzteblatt, 2002)

\section{Diagnosekriterien für ein langes QT-Syndrom}

(in Klammern die gewichteten Punktzahlen):

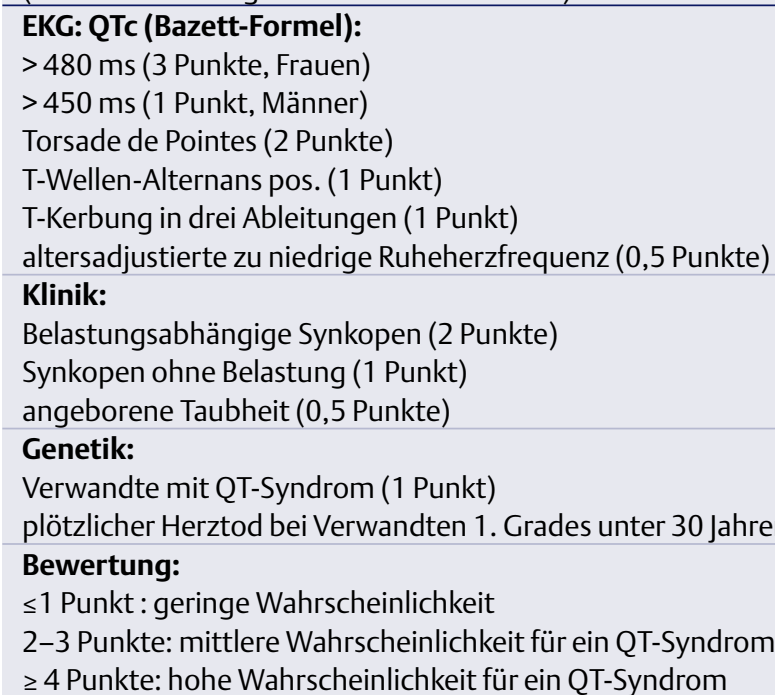

(Quelle: Schwartz et al., (27))

Zusammenhang darauf hin, dass der Anamnese - speziell der Familienanamnese, eine ganz besondere Bedeutung zukommt. Ein Kollaps bei jugendlichen Sportlern bedarf immer einer sorgfältigen Abklärung (Tab. 8, Tab. 9).

Bei der klinischen Untersuchung ist auf ein Marfansyndrom zu achten sowie auf mögliche Vitien. Die Aus-

\section{Tab. 6 Diagnostische Hinweise zum Marfansyndrom}

Kardial:

- Dilatation der Aorta ascendens

- Aortendissektion

- Aortenklappeninsuffizienz

- Mitralklappenprolaps

- Myxödematöse Mitralklappe

- Bauchaortenaneurysma

- Periphere Gefäßdissektion

Auge:

- Ektope Linse

- Flache Kornea

- Elongierter Augapfel

- Retinaablösung

- Myopie

Muskel/Skelett:

- Brustdeformität (Trichter- oder Hühnerbrust)

- Unproportional lange Finger, flache Füße

- Deformierungen der Wirbelsäule

- Große Körperlänge (im Vergleich zur Familie)

- Überdehnbare Gelenke, Gebissstörungen

Haut:

Striae, Hernien

Lunge:

Spontanpneumothorax

ZNS:

Ektasie der Dura

(De Paepe et al.: Am J Med Genet 1996; 62: 417-426, dort Auflistung der „Major and minor symptoms“)

\section{Tab. 7 Medikamente, Drogen und kardiale Komplikationen im Sport}

- Kokain Angina pectoris, Herzinfarkt, Kardiomyopathie

- Crack Rhythmusstörungen, Lungenödem u.a., Veränderungen an Lunge, Kopf, Hals, neurologische und psychiatrische Befunde

- Alkohol Rhythmusstörungen, Blutdrucksteigerung etc.

- Psychopharmaka

- Amphetamine

- Anabole Steroide

- Diuretika, Analgetika, Marihuana, Nikotin/Rauchen 


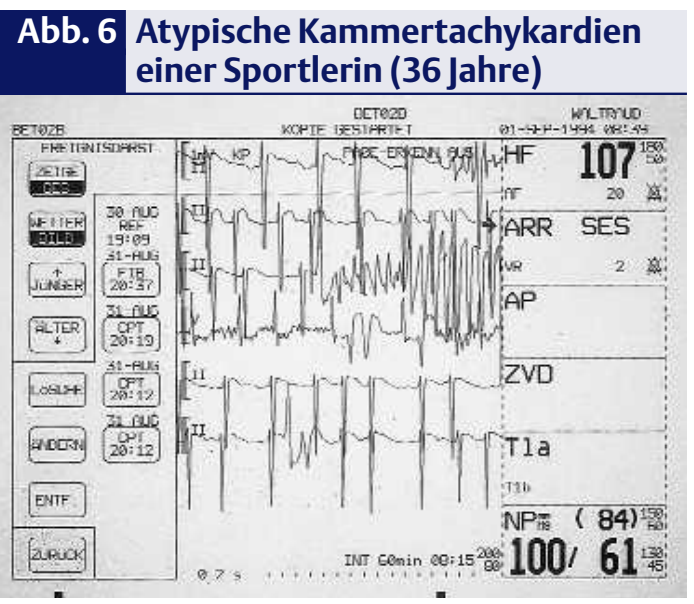

Atypische Kammertachykardien nach Einnahme von Terfenadin (Teldane ${ }^{\circledR}$ ) wegen Heuschnupfens. Es kam zum Herz-Kreislaufstillstand mit erfolgreicher Reanimation. Im Ruhe-EKG deutliche QT-Verlängerung.

kultation des Herzens muss im Liegen und im Sitzen erfolgen, da häufig nur dann Hinweise auf einen Mitralklappenprolaps oder eine HOCM gehört werden können.

$\mathrm{Zu}$ den apparativen Untersuchungen gehört heute obligat ein Ruhe-EKG, ein Belastungs-EKG ist wünschenswert. EKG und Belastungs-EKG setzen aber gute Kenntnisse voraus, um ein Brugada-Syndrom, eine ARVD-Erkankung, eine lange QT-Dauer oder ein mögliches WPW-Syndrom zu erkennen. Die Abgrenzung von sportbedingten Normvarianten im EKG gehört ebenfalls zum Standard. Das Belastungs-
EKG muss leitliniengerecht erfolgen und interpretiert werden. Häufigster Fehler ist die unzureichende Ausbelastung von Sporttreibenden. Im eigenen Krankengut finden sich einige Hochleistungssportler mit einer koronaren Herzkrankheit (hochgradige 1-Gefäßerkrankung), bei denen erst ab 250 Watt eine Ischämiereaktion zu beobachten war, der jeweilige Hausarzt hatte bei einer Belastung bis 125 Watt ein gesundes Herz bescheinigt (Abb. 5). Eine Herzechountersuchung sollte bei entsprechendem Verdacht (HOCM, offenes Foramen ovale etc.) durchgeführt werden. Es sei mit aller Zurückhaltung angemerkt, dass nicht jeder Kardiologe mit sportbedingten Herzveränderungen vertraut ist und nicht jeder Sportarzt spezifische kardiale Kenntnisse besitzt. Die Zusammenarbeit mit einem „kardiologisch versierten Sportarzt", beziehungsweise einem sportmedizinisch geschulten Kardiologen, ist bei schwierigen und speziellen Fragestellungen sinnvoll. Es ist darauf hinzuweisen, dass eine reine Vorsorgeuntersuchung nicht von den Kassen bezahlt wird, sie muss also als IGeL-Leistung abgerechnet werden. Inzwischen erstatten einige private Versicherungen solche Vorsorgeuntersuchungen. In den USA übernimmt der untersuchende Arzt die volle Verantwortung für etwaige Zwischenfälle bei Sportlern.

\section{Erstmaßnahmen bei kardia- len Zwischenfällen im Sport}

Bei kardialen Zwischenfällen im Sport gelten die gleichen Regeln und Empfehlungen wie bei sonstigen Notfällen. Beim plötzlichen Tod ist eine sofortige kardiopulmonale Reanimation nach den aktuellen Leitlinien mit Beginn der ABC-Maßnahmen einzuleiten.

Der plötzliche Tod im Sport beruht meist auf einer bedrohlichen Herzrhythmusstörung. Als lebensgefährlich gelten:

- Kammertachykardien (sowohl typische als auch atypische)

- Kammerflattern und

- Kammerflimmern sowie selten

- WPW-Syndrom mit intermittierendem Vorhofflimmern.

Alle diese Arrhythmien lassen sich mit einer Defibrillation unterbrechen und meist beseitigen. Dies gilt auch für das Kammerflimmern bei Contusio cordis.

Nach heutigen Erkenntnissen hat bei malignen Arrhythmien auch im Sport - nur eine rasche Defibrillation mit einem automatischen Defibrillator Erfolg. Ein solches Gerät muss heute, ähnlich wie in den USA, in jedem Stadion, beziehungsweise in jeder Sportarena vorhanden sein. Die Kosten sind, im Vergleich zu den üblichen Umsätzen in einem Fußballstadion, gering. Einer dieser Defibrillatoren sollte immer in der Nähe des Spielfeldes

\section{Abb. 7 Maligne Herzrhythmusstörungen bei einem jungen Sportler (18 Jahre)}
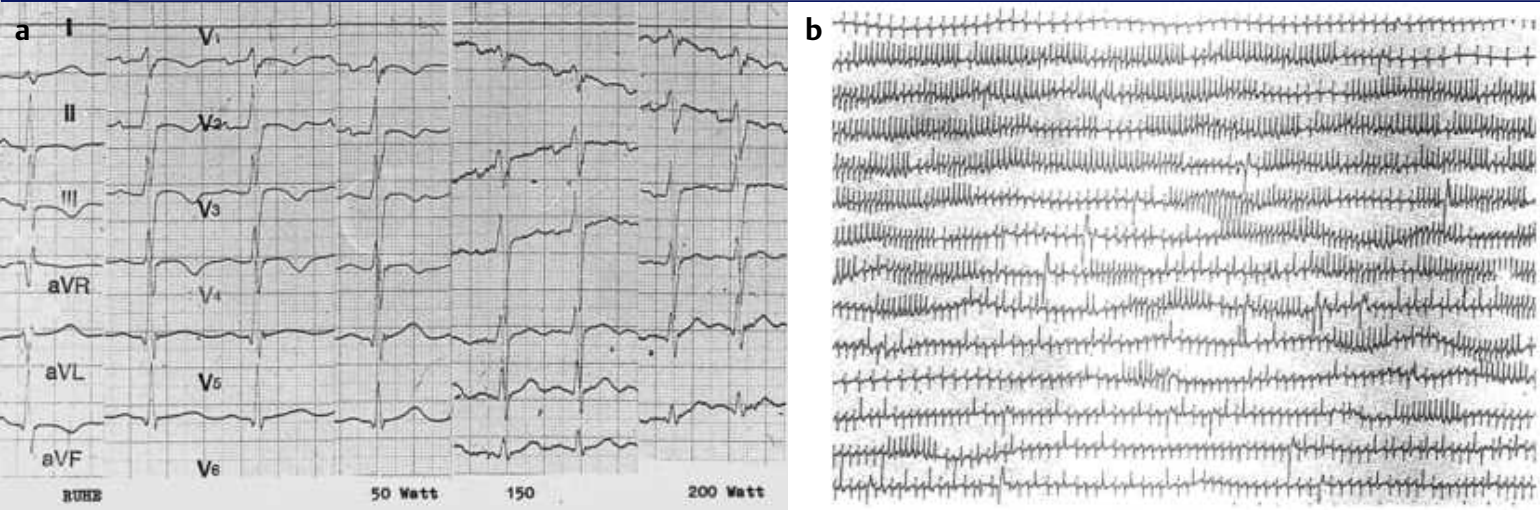

Maligne Herzrhythmusstörungen mit mehrfachen Synkopen. Im Ruhe-EKG (a) T-Negativierungen anteroseptal mit Verdacht auf ARVD. Im Belastungs-EKG (a) Aufrichten der T-Wellen. Im Langzeit-EKG (b) Torsade de pointes (atypische Kammertachykardien), typische Kammertachykardien, Salven und ventrikuläre Extrasystolen. Koronararterien unauffällig. Myokardbiopsie (Prof. Goodwin, London): Hypertrophe Kardiomyopathie. Unter Therapie mit $\beta$-Rezeptorenblockern beschwerdefrei. Nachbeobachtung über drei Jahre (Beobachtung von 1983). 
platziert sein (am Spielfeldrand). Der Mannschaftsarzt muss mit der Bedienung vertraut sein, desgleichen mindestens zwei weitere engagierte Laien. Grundsätzlich empfiehlt es sich bei größeren Sportarenen mehrere solcher Defibrillatoren anzubringen. Auch beim Training könnte eine tragbarer Defibrillator zur Ausrüstung der medizinischen Versorgung gehören. Man mag diese Forderung für ungewöhnlich halten, in einigen Ländern ist dies aber schon Routine. Im Vergleich zu den Bau- und Erhaltungskosten moderner Stadien spielen die Kosten für einen oder mehrere Defibrillatoren keine Rolle.

Problematisch ist es bei kleineren Sport- und Turnhallen. Hier ist der Kosten-Nutzen-Effekt ungünstig. Angesichts der geringen Wahrscheinlichkeit eines Zwischenfalls kann man hier auf einen Defibrillator verzichten. Finden allerdings in dieser Halle Sportangebote für Ältere oder für Patienten (Herzgruppe) statt, sollte heute ein rasch zugänglicher Defibrillator (Automat) vorhanden sein. So besitzen viele der modernen „Spaßbäder“ heute in aller Regel einen oder mehrere Defibrillatoren mit entsprechend geschultem Personal.

Auch bei allen größeren Sportereignissen wäre es empfehlenswert, einen tragbaren automatischen Defibrillator dabei zu haben.

Neben diesen Maßnahmen sollte natürlich der Notfallkoffer des Mannschaftsarztes oder der Mannschaft mit den wichtigsten Medikamenten zur Erstbehandlung kardialer Komplikationen, einschließlich der HerzLungen-Wiederbelebung (Adrenalin, Amiodarone, Magnesiumsulfat etc.), ausgestattet sein, neben der Ausrüstung für die Erstbehandlung von Verletzungen.

Hinzuweisen ist auf die Notwendigkeit, bei Sportlern - auch bei Berufssportlern - in regelmäßigen Abständen Kurse zur Reanimation durchzuführen. Ein Programm wie zur Führerscheinprüfung sollte bereits in der Schule bei 14- bis 16jährigen erfolgen; nach dem Führerschein in Sportvereinen alle zwei bis drei Jahre, bei Berufssportlern und Mannschaftsbetreuern ebenfalls alle

\section{Tab. 8 Abgestufte Diagnostik als Prävention im Sport}

\section{Abgestufte Diagnostik:}

- Fragebogen (standardisiert)

- Anamnesebogen (standardisiert)

- Körperliche Untersuchung (standardisiert)

- EKG in Ruhe (QT-Dauer) und während Belastung (standardisiert)

Nur bei speziellen Fragestellungen und verdächtigen Befunden:

- Langzeit-EKG (ggf. bis 72 Stunden), Tele-EKG über Wochen

- Herzultraschalldiagnostik (evtl. inclusive Kontrast- und transösophagealer Darstellung (Offenes Foramen Ovale?)

- Stressechokardiographie

- Kernspinuntersuchung bei Verdacht auf Myokarditis

- Kipptisch-Untersuchung

- Nicht gesichert bei Gesunden:

- QT-Dispersion

- Herzfrequenzvariabilität

- Chronotrope Funktion

- Spätpotentiale

- Barorezeptorenfunktion

- Herzkatheteruntersuchung: Links-, Rechtsherzkatheter

- Elektrophysiologische Untersuchung

\section{Tab. 9 Hinweise und Empfehlungen zur Vorsorge-} untersuchung Sporttreibender

\section{Vorgeschichte:}

- Belastungsabhängige Brustschmerzen/Unwohlsein, Synkope oder Beinahe-Synkope

- Belastungsabhängige vermehrte, unerwartete und nicht geklärte Dyspnoe

- Früher entdecktes Herzgeräusch

- Erhöhter Blutdruck (beide Arme, Beine)

Familienanamnese:

- Plötzlicher Herztod (vor allem bei jüngeren Familienmitgliedern) oder sonstige vorzeitige Herzkrankheiten

- Angaben zu Marfan-Syndrom, QT-Syndrom

- sonstige bedeutsame Arrhythmien

\section{Körperliche Untersuchung:}

- Unter anderem: Auskultation im Liegen und Stehen (!)

- Untersuchung der Femoralispulse, Suche nach Marfan-Zeichen

- Blutdruckmessung im Sitzen mit Angabe des Armes

- Routinemäßige Wiederholung alle 2 (?) bis 4 Jahre

- Bei Beschwerden und Symptomen: sofortige Untersuchung

(nach American College of Sports Medicine und American Heart Association,1998 sowie Empfehlungen der DGSP. Untersuchungsbögen unter dgsp@dgsp.de)

zwei bis drei Jahre ähnlich dem Programm der Luftverkehrsgesellschaften.

\section{- Prävention}

Neben der Schulung für Notfallmaßnahmen sollte aber der Prävention kardialer Zwischenfälle besondere Aufmerksamkeit gelten. Diese basiert auf einem sorgfältigen Vorsorgeuntersuchungsprogramm unter Beachtung der möglichen Risiko- faktoren (Tab. 8, Tab. 9). Ein generelles Screening aller Sporttreibenden ist derzeit aus Kostengründen nicht möglich und nach amerikanischen Untersuchungen auch nicht erforderlich $(17,18,21)$. Hilfreich wäre aber bereits ein Fragebogen nach dem kanadischen PAR-Q-Muster $(29,30)$. Sportärztliche Anamneseund Untersuchungsbögen werden von der Deutschen Gesellschaft für Sportmedizin und Prävention (DGSP, 
dgsp@dgsp.de) angeboten. Allen Sportlern aber, die eine Untersuchung wünschen, sollte diese angeboten werden. Die Abrechnung erfolgt nach den IGeL-Leistungen. Es sei aber angemerkt, dass auch bei noch so sorgfältiger Untersuchung eine absolute Sicherheit im Sport nicht gewährleistet werden kann.

Abschließend bleibt festzuhalten, dass trotz möglicher Risiken im Sport der Nutzen bei weitem überwiegt. Maron $(18,19)$ hat bei der Auswertung aller Marathonläufen in den USA (über 90 Stück) in einem Jahr festgestellt, dass die Zahl der Zwischenfälle unter den Teilnehmern geringer war als die Todesrate in der Allgemeinbevölkerung. Sport - mit Vernunft begonnen, mit Aufbautraining unter Anleitung und mit regelmäßigen sportärztlichen Untersuchungen $\mathrm{ab}$ einem Alter von 35 Jahren - ist eine sichere Betätigung mit nachgewiesenem Nutzen für Lebensqualität, Gesundheit und Lebensdauer.

\section{Summary}

Sudden cardiac death in sports is rare but a spectaculous and emotionally disturbing event. New cardiac arrhythmogenic diseases can explain sudden death which otherwise have been declared idiopathic. In younger athletes, structural and inborne heart disease prevail, whereas in elderly, those over 35 years, coronary artery disease is the predominant cause of sudden cardiac death even in athletes. With the advent of automatic defibrillators, successful treatment of sudden death even in the arena is possible, also in the hands of trained laymen. Prevention should be stressed more than before to avoid sudden death. A careful and standardized anamnesis is the mainstay, completed by a clinical examination with regard to special sports medicine aspects to further reduce the risks in athletes.

\section{Literatur}

1. Ahmad F, Duangxiang L, Karibe A et al. Localization of a gene responsible for arrhythmogenic right ventricular dysplasia to chromosome 3p23. Circulation1998; 98: 2971-2975

2. Alings M, Wilde A. „Brugada“ syndrom. Circulation 1999; 99: 666-673
3. *Balady GJ, Chaitman B, Driscoll, D et al. Recommendations for cardiovascular screening,staffing, and emergency policies at health/fitness facilities. Med Sci Sports Exercise 1998; 32: 1009-1018

4. Corado D, Basso C, Thiene G. Does sports activity enhance the risk of sudden death in young people? J Am Coll Cardiol 1999; $33: 119 A$

5. Corrado D, Basoo D, Thiene G. Sudden death in the athlete: The European perspective. In: Estes NAM, Salem DN, Wang PJ (eds.): Sudden cardiac death in the athlete. Futura Publishing Comp Armong, NY 1998; 285-300

6. *Estes NAM, Salem DN, Wang PJ (eds.): Sudden cardiac death in the athlete. Futura Publishing Comp. Armong, NY, 1998

7. Frohlich E. Left ventricular hypertrophy and sudden death. J Am Coll Cardiol 1998; 32: 1460-1462

8. Gussak I, Antzelevitch C, Bjeregaard P, Towbin JA, Chaitman BR. The Brugada syndrom: Clinical electrophysiologic and genetic aspects. J Am Coll Cardiol 1999; 33 : 5-15

9. Haider AW, Larson MG, Benjamin EJ, Lewy $D$. Increased left ventricular mass and hypertrophy are associated with increased risk for sudden death. J Am Coll Cardiol 1998; 32: 1454-1459

10. Link MS, Wang PJ, Pandian NG. An experimental model of sudden death due to low-energy chest-wall impact (commotio cordis). New Engl J Med. 1998; 338: 18051811

11. Löllgen $\mathrm{H}$, Hust MH, Nitsche K et al. Arrhythmogene Antiarrhythmika. Cardiology 1983; 70: 129-137

12. Löllgen H, Dirschedl P. Die kardiovaskuläre Gefährdung im Breitensport. Dtsch Z Sportmed 1989; 40: 212-221

13. Löllgen H, Bretschneider, Dirschedl P. Rhythm disturbances and exercise testing. In: Bachl N, Graham T, Löllgen H: Advances in Ergometry. Springer, Berlin 1991; 21-23

14. * Löllgen H, Erdmann E. Ergometrie. Springer, Heidelberg 2000

15. Maron BJ, Poliac LC, Kaplan AJ, Muller FO. Plunt impact to the chest leading to sudden death from cardiac arrest during sporting activities. New Eng J Med 1995; 333: 337342

16. Maron BJ, Thompson PD, Puffer JC et al. Cardiovascular Screening of competitive athletes. Med Sci Sports Exercise 1996; 30: 1445-1452

17. * Maron BJ, Mitchell JH (eds.). Recommendations for Determing eligibility for competition in athletes with cardiovascular abnormalities (26th Bethesda Conference). J Am Coll Cardiol 1994; 24: 845-899

18. Maron, BJ. Cardiovascular risks to young persons on the athletic field. Ann Intern Med 1998; 129: 379-386

19. Maurer MS, Shefrin EA, Fleg JL. Prevalence and prognostic significance of exercise - induced supraventricular tachycardia in apparently healthy volunteers. Am.J. Cardiol 1995; 75: 788-792

20. Meinertz T, Zehender M. Belastbarkeit bei Herzrhythmusstörungen ohne strukturelle Herzkrankheit. Dtsch Z Sportmed 1996; 47: 167-171
21. Murry, PM, Cantwell JD, Heath DL, Shoop J. The role of limited echocardiography in screening athletes. Am.J.Cardiol 1995; 76: 849-850

22. Myerburg RJ, Mitrani R, Interian A, Castellanos A. Identification of risk of cardiac arrest and sudden death in athletes. In: Estes NAM, Salem DN, Wang PJ (eds.): Sudden cardiac death in the athlete. Futura Publishing Comp. Armong,NY 1998; 25-56 23. Pellicia A, Maron BJ, Culasso $F$ et al. Clinical significance of abnormal electrocardiographic patterns in trained athletes. Circulation 2000; 102: 278-284

24. Peters S, Götting B, Peters $H$, Thierfelder L. Pathologie und Diagnostik der arrhythmogenen rechtsventrikulären DysplasieKardiomyopathie. Dtsch Ärztebl 1998; 95 : A1726-A1731

25. Samitz G, Mensink G (Hrsg.). Körperliche Aktivität in Prävention und Therapie. Marseille Verlag, München,2002

26. Schulze-Bahr E, Wedekind H, Haverkamp W et al. The LQT-syndroms - current status of molecular mechanisms. Z Kardiol 1999; 88: 245-254

27. Schwartz P, Moss AJ, Vincent GM, Crampton RS. Diagnostic criteria for the long QT-Syndrom. Circulation 1993; 88: 782-784

28. Shen WK, Edwards WD, Hammill SC et al. Sudden unexpected nontraumatic death in 54 young adults: A 30-year populationbased study. Am J Cardiol 1995; 76: 148152

29. * Shephard RJ, Cox MH, Simper K. An analysis of the „Par-Q“ responses in an office population. Can J Public Health 1981; 72 : 37-40

30. Thomas S, Reading J, Shephard RJ. Revision of the physical activity readiness questionnaire (PAR-Q). Can J Spt Sci 1992; 17: 338-345

31. Wilde AAM, Antzelevitch C, Borgrgefe M, Brugada J, Brugada R, Brugada P et al. Proposed diagnostic criteria for the Brugada syndrom. Circulation 2002; 106: 2514-2519 32. *Williams RA (ed.). The athlete and heart disease. Lippincott, Williams, Wilkins, Philadelphia, 1999

33. Zehender M, Meinertz T, Keul J, Just $\mathrm{H}$. ECG variants and cardiac arrhythmias in athletes: Clinical relevance and prognostic importance. Am J Cardiol 1990; 119: 13781391

34. Zimetbaum P, Josephson ME. Evaluation of patients with palpitations. New Engl ] Med 1998; 338: 13469-1373

(Die mit * gekennzeichneten Literaturstellen enthalten zahlreiche weiterführende Literaturangaben, ggf. weitere Literaturangaben bei den Autoren).

\section{Anschrift für die Verfasser}

Prof. Dr. med. Herbert Löllgen, FACC

European Cardiologist

Sana-Klinikum GmbH

Med. Klinik I

Burgerstr. 211

D-42859 Remscheid

E-mail: herbert.loellgen@gmx.de 